\title{
EMBEDDING E-RESOURCES FOR ACTIVE LEARNING
}

\section{A COLLABORATIVE PEDAGOGICAL MODEL FOR LARGE CLASSES}

Glenda Myers

\section{INTRODUCTION: INFORMATION LITERACY IN THE ERA OF LARGE CLASSES}

The creation of a learning culture that produces graduates with a capacity and desire for lifelong learning presumes a fundamental need for the acquisition of information literacy (IL) skills (Orr, Appleton \& Wallin 2001). IL underpins both the acquisition of knowledge and the ability to use that knowledge to function in all areas of an informed and developed society. As such, it is recognised as the overall critical literacy for the 21 st century (Bruce 2002). IL skills may be defined as a core set of competencies required to access, evaluate, organise and use information in order to learn, solve problems, and make decisions in formal and informal learning contexts (ACRL 2000). Consequently, the significance of IL in higher education cannot be underestimated, reaching far beyond the classroom to the lifelong ability to participate actively in a global information society.

At the same time, higher education currently faces unprecedented challenges arising from the convergent impacts of globalisation, the rapid pace of change in the information and communications environment, and the importance of knowledge as a principal driver of development and growth (World Bank 2002). At the same time, shrinking funds and the growing numbers of programmes offered in an attempt to offset budget cuts in the international higher education sector have seen unparalleled growth in class size, which further serves to exacerbate the challenges facing educators. While the difficulties faced by educators of large classes are well documented (Faculty Focus nd; Merlot Pedagogy 1997-2012; University of Maryland 2008), little mention is made of the importance of IL, or of the challenges of imparting this fundamental set of skills to students. The difficulties inherent in teaching IL skills to large classes are addressed even more seldom.

How best to achieve the aim of imparting IL skills when dealing with large classes is a question which deserves examination, especially in view of the increasing availability of web-based and open information sources for students, and their corresponding inability to assess and evaluate these sources within the context of their learning in the 
various subject disciplines. The need for IL skills has been intensified by the advent of Web 2.0 and the availability of open-content publications, which have significantly increased the amount of information available to students. As a result, there is increasing concern within higher education that students lack the competencies to assess these sources in terms of relevance to their courses (Barnes, Marateo \& Ferris 2007; Katz \& Macklin 2007; Shanahan 2009) or to process the information obtained cognitively (Walton \& Archer 2004). The way in which information is sought and interpreted is critical to the higher order cognitive levels in Bloom's Taxonomy of cognitive skills (Keene, Colvin \& Sissons 2010), and there are definite cognitive aspects involved in the information-seeking process itself (Kuhlthau 1991).

Unfortunately, educators do not always comprehend their students' underlying lack of IL skills and competencies. The increase in class sizes at universities does little to assist educators to analyse the problem. Instead, complaints abound that students 'copy and paste' material from the web and seem unaware of the ethical issues surrounding plagiarism (Renard 2000). The link between a lack of understanding about what constitutes plagiarism and IL skills is seldom explicitly stated.

In South Africa, where very large class sizes in higher education are becoming the norm, the internationally recognised problem of poor IL skills is intensified by the lack of a general culture of reading and libraries (Fairhead 2010). It is further exacerbated by the fact that English is not the predominant first language of most students at universities in this country, as well as by the poor primary and secondary schooling received by many students from disadvantaged backgrounds (De Jager \& Nassimbeni 2002).

\section{ACADEMIC LIBRARIAN INVOLVEMENT IN TEACHING IL SKILLS IN HIGHER EDUCATION}

When educators are alerted to a lack of IL competencies among their students as a major underlying cause of poor written work, they often lack the time or resources to deal with the problem as part of their teaching (McGuinness 2006). There is a widespread assumption that students will develop IL abilities simply by being sent to the library to make use of its resources (Badke 2005), or that they will somehow assimilate IL skills by virtue of their own "personal interest, individual motivation and innate ability" (McGuinness 2006:577. While the use of teaching assistants (TAs) is an acknowledged strategy for dealing with large classes (University of Maryland 2008), incorporation of the academic librarian's IL expertise into the formal curriculum to assist with students' IL skills development is seldom considered, even where large classes would indicate that such assistance might be welcome or even necessary. The term 'academic librarian' is used in this context to denote those librarians working in the higher education sector, as opposed to those in school libraries or in the corporate sector.

The vast body of literature confirms that effective collaboration between educators and librarians is still neither routine practice (Badke 2005; McGuinness 2006) nor widespread across subject disciplines, with the possible exception of medicine and 
nursing (Barnard, Nash \& O'Brien 2005; Brown \& Nelson 2003; Eldredge 1993; Jacobs, Rosenfeld \& Haber 2003; Miller, Jones, Graves \& Sievert 2010). The creation of a learning culture in which critical thinking and reasoning is the norm requires radical shifts in the educational paradigm (Orr et al 2001), and the strong focus on problem- and evidence-based learning in the disciplines of medicine and nursing probably explains why there is greater educator-librarian collaboration in this field than is seemingly the case in other subject disciplines. Collaborative effort by systematic review writing teams (incorporating both clinical expertise and library expert search expertise) in the health sciences has also likely helped to create a culture of trust and a shared language (Phelps \& Campbell 2012). In such a culture, all parties share common goals and are able to collaborate effectively and satisfyingly in this mutual endeavour (Swinkels, Briddon \& Hall 2006). However, problem-based learning (PBL) is typically used within small groups, and the issue of how to create this active learning environment when dealing with large classes has not been addressed within the context of IL skills development. Similarly, the difficulties of effectively imparting information retrieval and analysis skills (a core IL concept) to large groups are seldom discussed.

Although there is some evidence to suggest that the situation is beginning to change (Bewick \& Corrall 2010; Swinkels et al 2006), the link between IL skills and the evaluation of information found on the Internet, and the academic librarian's expertise in understanding how the literature is evaluated and synthesised, is mostly still not explicitly recognised in higher education.

\section{TENSIONS IN IL SKILLS COLLABORATION}

The fact that more attention has not been paid to IL skills from a pedagogical perspective may be due to librarians not having been proactive enough in promoting the benefits of collaborative teaching (Stevens 2007). Librarians have also possibly not participated greatly in faculty development programmes and 'train-the-trainer' outreach programmes where teachers are taught about IL skills and how they may be incorporated successfully into various discipline-specific curricula (Jirojwong \& Wallin 2001; Miller, O'Donnell, Pomea, Rawson, Shepard \& Thomes 2010).

Librarians do not tend to publish much in disciplinary journals other than those in their own field of library and information science (Hardesty 1995; Still 1998). Stevens (2007) argues convincingly that the transferability of general teaching concepts and strategies, in addition to the problem-based focus of many of these pedagogical journals, make them a natural platform for librarians to reach out to faculty and promote IL within the subject-specific disciplines. While educators are often disconnected from the process of student research, seeing only the final research product and the problems inherent in these papers, librarians deal with the common day-to-day problems that students grapple with in terms of their lack of information skills. These problem-based case studies might often be candidates for publication in the pedagogical literature, if the issue of a common language to describe the same processes could be overcome (Gullikson 2006). 
A lack of IL skills among teachers themselves is suggested as a possible reason for the difficulty in integrating these skills into content teaching for their students (Kong 2007; Stevens 2007). Therefore, the substantial barrier to faculty-librarian collaboration may not only be associated with the already mentioned lack of a common language to describe the same process, but may also be linked to teachers' lack of internalisation of the role of information in their own professional learning. If there is a tension between IL theory and the real-world information experience of teachers, this may translate into difficulties in modelling good practice for their own students (McGuinness 2006; Oker-Blom 1998; Stevens 2007; Williams \& Coles 2007). When teachers' own IL skills and their information-gathering methods were examined, it was found that informal methods of information gathering (such as personal contact and communication) for both teaching and research were relatively common among those educators who did not feel confident about their own more formal IL skills - skills that they expect their students to have. Such educators were also not comfortable about asking questions regarding sources of information, particularly the newer electronic resources. Information overload was also suggested as a factor in the lack of more formal information-gathering methodologies (Jirojwong \& Wallin 2001).

Another tension occurs as a result of apparent 'infiltration' by librarians who, to a large extent, have not been considered legitimate members of the subject faculties, even when they have been granted that official status. The consequent unease among librarians, university administrators and conventional teaching staff remains, for the most part, a hurdle to be overcome (Owusu-Ansah 2004). Doubt is expressed about the importance of the teaching role of librarians within many subject disciplines, possibly as a result of sensitivity regarding the professional autonomy afforded by the position of many subject-specialist educators within the institutional or faculty culture as a whole (Hardesty 1995; McGuinness 2006).

Despite recognition of the value of IL skills by educators at one United Kingdom-based university, it was noted that there was very little activity on the part of academic staff to either teach or assess these skills, or to develop programmes through studentcentred learning activities (Weetman 2005). Qualitative analysis of comments made by academics from a 'soft' discipline (sociology) and a 'hard' one (civil engineering) in another UK-based study show how entrenched disciplinary-based beliefs and perceptions may adversely affect the potential for collaboration and prevent the inclusion of IL skills in undergraduate curricula (McGuinness 2006).

Not much agreement could be found in the literature about the academic level at which IL outcomes are expected by educators. Where there was agreement, it tended to be with regard to outcomes expected in the first year of university (Gullikson 2006). This might explain the emphasis of many first-year programmes in the higher education sector internationally in which IL competencies play a part, such as general 'First-Year Experience' skills programmes, or the targeting of first-year writing courses and other large first-year classes. However, the variation between different disciplines with regard 
to how IL is conceptualised by academics largely negates the notion that all IL skills are somehow generic and transferable, and that they may therefore be taught as a 'stand-alone' course (Grafstein 2002; Owusu-Ansah 2004). Germane to the debate about the 'generic versus situated' nature of IL skills, students' experiences of IL led to the conclusion that while some aspects of IL are generic, others are context-specific to the subject discipline (Lupton 2008). There is also little conceptualisation regarding the different levels of IL required in first-year courses, for example, as opposed to the in-depth IL skills required by the senior postgraduate student.

Information literacy at the primary and secondary school levels falls outside the scope of this discussion, but, interestingly, the impression gained from the literature is that it appears to be largely accepted for the school librarian (or media specialist), particularly in the North Americas, to work together with the classroom teacher to create space for these skills within the curriculum itself, despite wide fluctuations from state to state in the standard of IL instruction in USA schools. Therefore, unlike in the higher education sector, collaboration between teaching staff and librarians at schools seems to be the norm, rather than the exception (Sanborn 2005; Taylor 2006). There is widespread recognition in the USA, especially at secondary school level, that teaching IL skills constitutes an important component of educational success (Smalley 2004). As such, most secondary schools have their own libraries, usually with a professional librarian in charge (Osborne 1989). Sadly, in South Africa, only eight per cent of schools reportedly have their own libraries, and the South African government has argued that the campaign for 'one library, one school, one librarian' is not financially viable (Fairhead 2010). The result of this situation may be seen in the dearth of IL skills among both educators and students in South African higher education.

\section{COLLABORATIVE IL TEACHING IN HIGHER EDUCATION}

A 2002 overview of the state of information literacy in higher education in South Africa demonstrated little integration of IL skills into subject curricula locally (De Jager \& Nassimbeni 2002). Internationally, there has been some evidence in pedagogical journals and discipline-specialist education literature of collaboration between educators and librarians in teaching IL skills during recent years (Brasley 2008; Floyd, Colvin \& Bodur 2008; Freeman \& Lynd-Balta 2010; Mackey \& Jacobson 2005; Stevens \& Campbell 2008). This partnership is noted particularly with regard to writing skills courses, where language composition teachers are described as the natural partners of librarians in seeking to develop robust and effective IL programmes (Hlavaty \& Townsend 2010). Collaboration between faculty and librarians to facilitate scientific writing also receives some attention (Jacob \& Heisel 2008). It is noted that changing teacher practice in order to incorporate IL skills into the classroom requires not only recognition of its value, but also the motivation to do so (Hara 2006). In this respect, meeting accreditation standards seems to be a particularly strong motivation for educators to partner with librarians (Birch, Greenfield, Janke, Schaeffer \& Woods 2008). 
There is some speculation that the concept of IL competencies needs to fit with institutional culture before it will be accepted (Tyron, Frigo \& O'Kelly 2010), and that pedagogic knowledge (in addition to discipline-specific knowledge and LIS qualifications) is required of all librarians who teach (Bewick \& Corrall 2010; Mestre, Baures, Niedbala, Bishop, Cantrell, Perez \& Silfen 2011). Both departmental and institutional culture is thought to play a major role in the readiness of an institution to accept IL programmes as part of a more holistic approach instead of the isolated 'silo' model, where IL skills are seen as peripheral (rather than critical) to learning outcomes. The move towards student-centred approaches of learning (as encapsulated in problem and enquirybased models of learning) in many institutions of higher education, as well as greater emphasis on interdisciplinary and cross-modular studies, thus holds potential for greater integration of IL programmes into the various curricula (Travis 2008).

\section{INFORMATION LITERACY IN HEALTH SCIENCES CURRICULA}

Academic beliefs and perceptions about how IL skills are conceptualised appear to be different in the field of the health sciences, as compared to other disciplines. This may, in part, be due to the fact that situated skills training plays such a large role in clinical teaching. The need to teach evidence-based practice (EBP) has also made educators in the health sciences more cognisant of the requirement for IL skills and competencies to be integrated into clinical teaching, and more willing to delegate the teaching of these skills to medical librarians. In the health sciences, EBP requires that clinical knowledge and skills be consciously integrated with an ability to search the literature in order to retrieve the evidence needed to support a clinical decision. The acquired information must subsequently be critically assessed for validity, reliability, and appropriateness, according to specific evidence-based medicine (EBM) criteria, in order to apply the evidence to patient care (Rosenberg \& Donald 1995). Thus the IL skills required of medical students must go beyond digital searching and retrieval to include the contextualisation, analysis and synthesis of medical information. These are complex skills that require critical thinking (Lorenzo \& Dziuban 2006). Information literacy skills are therefore of critical importance in teaching medical students to engage with EBM, often within a PBL curriculum (Gruppen, Rana \& Arndt 2005; Kingsley \& Kingsley 2009). The interactive nature of EBM, where clinical knowledge must be synthesised with the retrieval and analytical skills required to find and evaluate the evidence in the literature, suggests that the health sciences are a natural arena for teaching partnerships between clinical teachers and librarians. The teaching of evidence-based information-seeking skills, as well as teaching assessment and evaluation methodologies for evidence-based practice, has thus afforded opportunities for the librarian to become a partner in the health care process (McKibbon \& Bayley 2004; Swinkels et al 2006). The librarian also often assists with problem-based tutorial facilitation within EBP (Eldredge 2004).

Medical librarians were among the forerunners in introducing IL initiatives (Brown \& Nelson 2003; Burrows, Ginn, Love \& Williams 1989; Burrows \& Tylman 1999). These 
early adopters were no doubt influenced by the need to provide IL skills to students engaged in PBL activities, where the focus on student-centred learning emphasises active learning techniques (Eldredge 1993; Fitzgerald 1996; Marshall, Fitzgerald, Busby \& Heaton 1993; Oker-Blom 1998; Rankin 1996; Rankin 1992), a development which, in turn, was almost certainly the result of the adoption of this form of instruction by many medical education programmes internationally, following its introduction into the medical curriculum at McMaster University in the 1960s (Norman \& Schmidt 1992).

The teaching of EBM saw some early and ongoing collaborative initiatives between medical librarians and medical educators, as the need for specific EBM information literacy skills became imperative (Dorsch, Aiyer \& Lynne 2004; Vogel, Block \& Wallingford 2002). Despite a suggestion that the rise in popularity of many PBL-based medical curricula may be ascribed to a concurrent explosion in the biomedical literature (Epstein 2004), very few studies on IL skills teaching could be found in journals relating to clinical medical education (Hamilton 2008; Morris \& McKimm 2009; Oberprieler, Masters \& Gibbs 2005; Shanahan 2009). Interestingly, the nursing (as opposed to medical) literature contains far more examples of curriculum-integrated IL skills and faculty and library partnerships (Barnard et al 2005; Innes 2008; Jacobs et al 2003; Klem \& Weiss 2005; Miller et al 2010; Phillips \& Bonsteel 2010; Schutt \& Hightower 2009; Shorten, Wallace \& Crookes 2001; Verhey 1999). The reason for such differences in subject-domain collaboration within the health sciences would form a topic for further research.

It may be that medical educators' lack of commitment to embedding IL skills into the curriculum is due, in part, to the need for professional development in terms of improving clinical teachers' own IL skills (Williams \& Coles 2007). It may also be that medical educators require professional development in order to incorporate an understanding of evidence-based information seeking into their own teaching practice (Ulvenes, Aasland, Nylenna \& Kristiansen 2009).

Notwithstanding the paucity of publications on collaborative IL skills teaching in the medical literature, a 2011 survey of educators involved in the Graduate Entry Medical Programme (GEMP) curriculum at the Faculty of Health Sciences of the University of the Witwatersrand (Wits), Johannesburg, revealed no existing tensions between educators and librarians with regard to in-curriculum teaching of IL skills (Myers 2011). The majority of respondents to this survey (sixty-nine point four per cent $(69.4 \%)$ ) indicated that librarians, in conjunction with both medical practitioners and pre-clinical scientists, should be involved in co-teaching IL skills to students in this faculty, while a further twenty-one per cent thought that librarians together with medical practitioners (but not pre-clinical scientists) should be responsible for such teaching. This finding may be a result of the integrated nature of teaching across all subject disciplines in this programme, as well as the fact that the use of evidence from the literature for lifelong learning and continued professional development is a stated outcome of the GEMP in this faculty. 


\section{EMBEDDED INFORMATION LITERACY}

If students are to learn from the vast quantity of digital resources available to them, IL skills development cannot stand in isolation from the context of subject material. IL skills need to be woven into the learning experience itself (ACRL 2000; Bruce 2002), and not taught extraneously to subject content. When IL skills are taught within such a collaborative framework, the link between student-centred active learning and higher order cognitive skills (such as critical thinking) is effectively demonstrated and clear (Keene et al 2010). Embedded IL skills are more likely to produce transferable learning and cognitively processed knowledge (Walton \& Archer 2004), as opposed to mere recycling of information. From the perspective of the student, who is as yet not cognisant of the overarching nature of this skill set, the segregation of fundamental IL skills from the teaching process itself is exaggerated when IL skills are taught in isolation from the curriculum. There is little understanding on the part of students that library resources must be integrated into their subject discipline knowledge, or of how this might be achieved. Simply setting a reading list, and assuming that students will indeed both read and absorb knowledge from the material on this list, is not sufficient (Stokes \& Martin 2008). Nor, unfortunately, will students assimilate IL skills simply by visiting the library or using it as a place to study (Badke 2005).

In addition to addressing the problems inherent in teaching large classes, the overall approach to teaching and learning needs to evolve in order to meet the needs of "Net Generation" learners (Sandars \& Morrison 2007:85). These learning needs include a rich multimedia environment, including audio and visual images. Active engagement in tasks is preferred to reading or writing about events, and immediate feedback is expected. Motivation to learn comes from being actively involved in the learning process and attempting to answer questions that arise during the process of completing the tasks that are set (Oblinger \& Oblinger 2005). In this regard, the web offers a rich teaching and learning medium that is particularly suitable for use with large classes. However, in this context it must be clearly understood that it is not technical skills that make for effective web users, but rather the "reflective and conceptual capabilities that are part of the character of the information literate" (Bruce 2002:15).

While curriculum practices involving active collaborative resource-based learning are often seen as the ideal, they are still fairly innovative. There is a need to move away from the "dominant paradigm of prepackaged information" for students - in the form of textbooks and course or reading packs - to more active learning modalities (Bruce 2002:12), and the academic librarian is well able to assist in this collaborative endeavour. There is a fundamental lack of understanding as to the educational role of the librarian, as distinct from the 'warehouse manager' of the resources stored in the physical library (McGuinness 2006). Contrary to educators, who are often disconnected from this process, librarians work with students engaged in research and information seeking on a day-to-day basis (Stevens 2007). As such, educators should consider librarians as an effective teaching resource, instead of simply referring students to them 
to find material that is housed in the physical (or even virtual) library itself. However, a relationship based on trust and shared goals, as well as a common language to describe these goals, may need to develop before educators are willing to use the academic librarian as an additional resource for teaching IL skills (Gullikson 2006; Phelps \& Campbell 2012; Swinkels et al 2006).

\section{DYNAMIC PURPOSEFUL LEARNING}

Commitment on the part of educators to embedding IL skills in the curriculum may, in part, require an acceptable theoretical pedagogical framework into which collaborative teaching of IL skills may be inserted. Dynamic Purposeful Learning (DPL) (Kenedy \& Monty 2008) has been proposed as a collaborative framework for the introduction of IL skills practice into teaching, based on the idea that student learning is enhanced by collaborative teaching (Montiel-Overall 2006; 2008). DPL draws on various pedagogical strategies such as active learning, cognitive scaffolding and collaborative learning, where skills are connected to form and content is related to learning the subject matter. DPL is further informed by the idea of 'cognitive apprenticeship', where "activity, concept and content are interdependent" and "learning must involve all three" (Brown, Collins \& Duguid 1989:33). Cognitive apprenticeship is particularly suited to medical education, in that much of the clinical teaching takes place in the workplace (at patients' bedsides or in outpatient clinics) and much of the clinical learning is modelled on the behaviour of the clinical teachers.

Dynamic Purposeful Learning emphasises an active and ongoing partnership between the educator and librarian, starting with the development of the curriculum itself and the setting and assessment of integrated assignments throughout the course, and is seen in the classroom itself, where the "teaching dynamic" between educator and librarian is recognised by students as integral to the acquisition of situated knowledge in the subject discipline (Kenedy \& Monty 2008:89). The benefits of this collaboration are thus not restricted to the educator and librarian, but are understood in context by the students, who themselves form part of the learning dynamic generated by this collaboration. The collaborative dynamic can also enhance the educator's own internalisation of the process of becoming information literate as a student, thereby contributing to a deeper understanding of the extent of intervention and guidance required for IL skills to develop as part of a structured pedagogical approach (McGuinness 2006). The gap between librarian-centred and academic ways of thinking about IL skills is thus effectively bridged, and in this collaborative endeavour "student learning can be both supported and transformed" (Kenedy \& Monty 2008:92). DPL is thus presented as a pedagogical model based on three elements: an educator working with a librarian in the context of an assignment; purposeful learning that is applied to the assignment; and the assignment itself, which is completed in different stages. It is emphasised that a key element to the "dynamic" is the initial stage of contact with students, when the educator and librarian are in the classroom together (ibid:89). 
The DPL model holds promise for the integration of IL skills into the clinical medical curriculum using an adaptation of an e-learning roadmap (Morris \& McKimm 2009). This roadmap is devised from a model of complex strategic processes employed in moving the potential of e-learning in universities to a new level of development (Salmon 2005). Salmon notes that academic staff at universities are naturally reluctant to change existing methods of teaching without a thorough understanding of what benefits might accrue from such change. Variables that could influence the attitudes of academic staff towards the adoption of new practices include intellectual reluctance, a lack of change management programmes, a perceived lack of support for such change, and perceptions of a poor cost-benefit ratio (Mitchell \& Geva-May 2009). These perceptions would appear similar to those regarding the integration of IL skills into the curriculum (such as reluctance to cede time in the curriculum for formal teaching of IL skills).

Furthermore, given the research imperatives in most institutions of higher learning, there may be little time or desire to incorporate the new forms of constructivist knowledge sharing offered by many online learning technologies, unless the research evidence about online learning itself becomes embedded in the everyday teaching processes at a university. The early focus on technological infrastructure in e-learning seems to have given way to increased discussion about pedagogy and collaboration, merging newer approaches to learning with those used previously. The design of e-learning activities, such as the integration of IL skills into blended course content, might consequently come to assume more importance (Salmon 2005). The envisioned roadmap for the integration of IL skills into a medical curriculum (Morris \& McKimm 2009), supports the e-learning strategy suggested by Salmon (2005) in so far as the development and support of new models of good practice may be integrated into the everyday teaching practice of a medical curriculum.

\section{THE USE OF LIBGUIDES FOR COLLABORATIVE EMBEDDING OF LEARNING OBJECTS}

LibGuides are a web-based content management and knowledge-sharing system from the Springshare Corporation (Springshare Corporation 2010-2013), offering access to particular areas of study and enabling focused and scaffolded support for any subject domain. LibGuides are essentially learning objects, which may be described as small (relative to the size of the entire course) instructional components that may be reused in different learning contexts. Pedagogically sound instructional design for each LibGuide is required to facilitate effective learning (Mestre et al 2011). LibGuides can also be used extremely effectively to provide small chunks or bite-sized pieces of basic knowledge, such as definitions and background material, or even exercises for active learning. These starting points for students may easily be tailored to learning outcomes, and may also provide opportunities for critical thought and analysis if the LibGuide resources are linked to specific assignments (Miner \& Alexander 2010). In addition, LibGuides may be tailored to meet specific IL needs at different levels of study, so that 
the citations needs of students in a first-year course may be met as effectively as those of Master's students working towards a specialist medical qualification.

Each LibGuide affords the student a gateway with immediate hyperlinked access to information resources relevant to any particular course. Whereas attaching a full text article directly to e-learning management system (LMS) content does not support active learning, the LibGuide involves the student in interactively searching for relevant, guided sources of information, and is thus consistent with the principles of scaffolding. It is also possible to incorporate tools such as videos, blogs, news feeds, chat rooms and instant messaging, as well as screencast tutorials, links to academic databases, journal articles, and library catalogues and e-books. The interactive polling feature in LibGuides offers a much more cost-effective alternative to the use of clickers, as the poll is conducted online without the need for each student to have a clicker, thus removing the need to purchase clicker software. The effect of the interactive polling feature is exactly the same as that of using clickers, and it is suitable for classes where students have access to any handheld device and wireless fidelity (Wi-fi) technology.

LibGuides are ideal for promotion to students by both librarians and teaching staff, and may be set up in collaboration with the educator. If so required, the educator may provide input as a formal collaborator, and may actively contribute content to the LibGuide. It is noted that students will not necessarily use these guides unless they find them useful for completing assignments, in much the same way that they will not necessarily use recommended readings from a reading list (Stokes \& Martin 2008). However, experience at the Witwatersrand Health Sciences Library (WHSL) shows that its guides were extensively used, with 71,995 views generated from thirty guides in 2012.

An added advantage of LibGuides is that they can be evaluated by users, as can individual resources, thus offering a form of peer evaluation. These evaluations and ratings provide useful feedback to library and teaching staff for revision of the guides, where necessary.

\section{THE 'EMBEDDED LIBRARIAN' AND IL IN LARGE CLASSES AT THE WITS FACULTY OF HEALTH SCIENCES}

The Faculty of Health Sciences at Wits employs a hybrid mix of didactic and problembased learning in many of its curricula across the eight degree courses it offers. PBL is generally used in small groups, but ministerial pressure from the Department of Health to meet national developmental needs (an increase in the numbers of graduates in disciplines such as medicine) has led to an unprecedented growth in the size of classes over recent years, which is seldom accompanied by a corresponding increase in the number of staff to teach these classes. Nor is there necessarily an increase in suitable teaching venues. Large lecture classes in tiered amphitheatres do little to encourage active learning methods: discussion is hampered by the constraints of fixed seating in such large lecture halls, and other modalities for increased student participation are not always effective (Herreid 2006). 
Although PBL is deemed particularly suitable for curricula in the health sciences because of the emphasis on EBP, it is usually practised in small groups and is not suited to large classes. Repetition of the same material, as a strategy to cope with increased class sizes, gives rise to issues of consistency and quality assurance, as well as boredom and not infrequent burnout on the part of the same few individuals tasked with teaching these classes. The use of the 'embedded librarian' as a teaching assistant - either physically co-teaching in the classroom in terms of IL skills instruction, or virtually integrated into any LMS by means of a LibGuide presence - can be extremely effective in alleviating the teaching load in large classes. The use of the LibGuide ensures consistency in teaching, thus addressing quality issues. Each LibGuide has a unique universal resource locator (URL), so that virtual embedding into the LMS is easily accomplished, thus endorsing the DPL model for collaborative teaching.

One such WHSL guide for pharmacy and pharmacology at the Wits Faculty of Health Sciences has been embedded into the e-learning system used by lecturers in that department for 'anywhere, anytime' point-of-need access. It received over 8,000 hits in 2011 from two classes (Pharmacology III and Pharmacology IV), totalling approximately 140 students. Five other WHSL LibGuides are used to teach effective evidence-based information seeking and retrieval as part of the formal curriculum, incorporating IL competencies for two groups (GEMP 1 and GEMP 2) comprising approximately 500 medical students in the third and fourth years of the medical degree, and were collectively viewed a total of more than 7,000 times in 2011. As these LibGuides are available $24 / 7$ and are not housed on university servers, they can be accessed without impediment from any location and using any device capable of connecting to the Internet. As such, they can be used in the clinical teaching environment of the hospital ward. Medical students report using these resources at point of need to gain understanding of underlying concepts involved, find relevant e-resources for their studies and assignments, and revise for their information retrieval assessments and examinations. Despite very large class sizes, teaching staff report satisfaction with improved student performance in terms of their written work after library involvement in the co-teaching of IL skills. The use of embedded LibGuides has been instrumental in decreasing the amount of time required for one-to-one coaching, whilst still providing individualised scaffolding, as social media tools can be effectively incorporated into the LibGuides to provide virtual help when required.

Other instances reporting extremely high use of LibGuides by large classes are beginning to appear in the literature (Mestre et al 2011; Miner \& Alexander 2010). While programmes will require further research and evaluation to demonstrate conclusive effectiveness in terms of IL outcomes, the concept of collaborative teaching by librarians and faculty, using learning objects such as LibGuides, has provided a highly effective method of instruction for large-class teaching, both by incorporating IL skills into the curriculum and by providing additional scaffolding for students. 


\section{CONCLUSION: LINKS BETWEEN LARGE-CLASS TEACHING AND COLLABORATIVE EMBEDDED RESOURCES}

As early as 1998, Breivik noted that IL cannot be taught by librarians or faculty in isolation, but must rather be learned by students through experiences shaped by librarians and faculty working together (Breivik 1998). Information literacy, contrary to the views of some academic staff, cannot be acquired by serendipity in the same way that information itself can be gathered by browsing. The teaching of IL skills thus needs a strategic curricular and collaborative approach (Gullikson 2006; McGuinness 2006; Travis 2008).

At the same time, teaching large classes requires a repertoire of instructional strategies, and the decision of which strategy to use when will be guided by many variables. In the case of large-group teaching, collaboration with the academic librarian should be one of these strategies, especially where the development of IL skills is seen as a priority for an informed society. Whether teaching large classes in a collaborative and physical dynamic in the classroom alongside the subject specialist educator (such as in the DPL model), or having a virtual presence in the form of embedded learning objects in subject specialist LMS modules (such as with the use of LibGuides), the concept of the 'embedded librarian' has proved viable at the Wits Faculty of Health Sciences. In a climate where mutual trust, shared goals, a common language to describe these goals, and collaborative endeavour are developing and appreciated by all concerned, the daunting task of imparting IL skills to very large groups of students has been embraced, and has enhanced the ability of students in the health sciences to master a complex set of evidence-based IL skills. 